\title{
Demonstration of a GaSb/GaAs Quantum Dot Intermediate Band Solar Cell Operating at Maximum Power Point
}

\author{
I. Ramiro $\odot,{ }^{1, *}$ J. Villa $\odot,{ }^{1}$ J. Hwang $\odot,{ }^{2}$ A. J. Martin, ${ }^{3}$ J. Millunchick, ${ }^{3}$ J. Phillips $\odot,{ }^{2}$ and A. Martí ${ }^{1}$ \\ ${ }^{1}$ Instituto de Energía Solar, Universidad Politécnica de Madrid, 28040 Madrid, Spain \\ ${ }^{2}$ Department of Electrical Engineering and Computer Science, University of Michigan, Ann Arbor, Michigan 48109, USA \\ ${ }^{3}$ Department of Materials Science and Engineering, University of Michigan, Ann Arbor, Michigan 48109, USA
}

(Received 31 May 2020; revised 23 September 2020; accepted 3 November 2020; published 9 December 2020)

\begin{abstract}
Intermediate band solar cells (IBSCs) promise high efficiencies while maintaining a low device structural complexity. A high efficiency can be obtained by harvesting below-band-gap photons, thus increasing the current, while at the same time preserving a high voltage. Here, we provide experimental proof that below-band-gap photons can be used to produce nonzero electrical work in an IBSC without compromising the voltage. For this, we manufacture a GaSb/GaAs quantum-dot IBSC. We use light biasing and make our cell operate at the maximum power point at $9 \mathrm{~K}$. We measure the photocurrent response to absorption of photons with an energy of less than $1.15 \mathrm{eV}$ while the cell is operating at $1.15 \mathrm{~V}$. We also show that this result implies the existence of three quasi-Fermi levels linked to the three electronic bands in our device, as demanded by the IBSC theory to preserve the output voltage of the cell.
\end{abstract}

DOI: 10.1103/PhysRevLett.125.247703

The conversion efficiency of conventional solar cells is fundamentally limited by the fact that they rely on singlegap (SG) semiconductors as the absorbing material. The band gap of the SG material sets the upper limit for both the generated current and the output voltage of the cell. A lower band gap leads to higher current at the expense of lower voltage. The reverse occurs if the band gap is increased. This trade-off between current and voltage finds its maximum efficiency limit of around $41 \%$ for single-gap solar cells (SGSCs) operating at $300 \mathrm{~K}$ under maximum solar concentration [1]. Intermediate band solar cells (IBSCs), capable of achieving efficiencies as high as $63 \%$ [2], were proposed as an alternative to SGSCs. The IBSC relies on the so-called intermediate band (IB) semiconductors as the absorbing material of the solar cell. As detailed in Fig. 1(a), IB materials exhibit a third electronic band, the IB, in between the valence band (VB) and the conduction band (CB). The IB splits the main band gap, $E_{C V}$, into two sub band gaps, $E_{I V}$ and $E_{C I}$, thus enabling below-band-gap photon absorption via a sequential twostep process (transitions labeled 1 and 2) in addition to the absorption of photons with an energy higher than the band gap (transition 3). Analogously, the presence of the IB enables extra band-to-band recombination processes (labeled 1' and 2'). In an ideal IBSC delivering actual

Published by the American Physical Society under the terms of the Creative Commons Attribution 4.0 International license. Further distribution of this work must maintain attribution to the author(s) and the published article's title, journal citation, and DOI. power, the extra absorption outmatches the extra recombination, which results in an overall increased photogenerated current $J$. In the IBSC model, the output voltage $V$ is said to be preserved, meaning that it is fundamentally limited only by $E_{C V}$ (as is the case for SGSCs) and not by $E_{C I}$ or $E_{I V}$. The preservation of a high voltage is sustained by the existence of three distinct electrochemical potentials or quasi-Fermi levels (QFLs), $\mu_{I}, \mu_{V}$ and $\mu_{C}$, associated to the IB, the VB, and the $\mathrm{CB}$, respectively. In the IBSC model $e V=\left(\mu_{C}-\mu_{V}\right)$, irrespective of $\mu_{I}$, where $e$ is the (a)

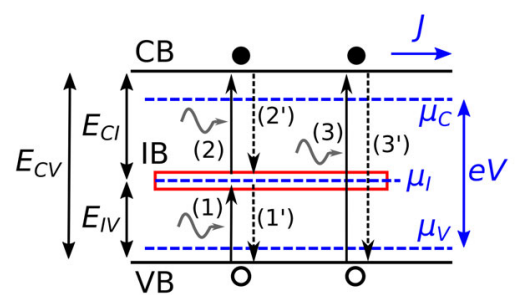

(b)

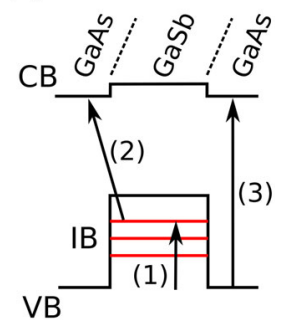

FIG. 1. (a) Simplified band diagram of the IB material in an IBSC under operation. The photocurrent originates from belowband-gap photon absorption (via transitions 1 and 2) in addition to above-band-gap absorption (transition 3). The corresponding three band-to-band recombination processes, $1^{\prime}, 2^{\prime}$, and $3^{\prime}$, are also indicated. Three QFLs, $\mu_{C}, \mu_{V}$, and $\mu_{I}$, are associated to the $\mathrm{CB}$, the $\mathrm{VB}$, and the $\mathrm{IB}$, respectively. The photogenerated current $J$ is delivered at a voltage $V=\left(\mu_{C}-\mu_{V}\right) / e$. (b) Simplified band diagram of an IB material implemented with $\mathrm{GaSb} / \mathrm{GaAs}$ QDs, exhibiting a type II (staggered) band lineup with confined hole states serving as the IB. For simplicity, only three confined states have been indicated. 
elementary charge. By delivering a higher current while maintaining a high output voltage (greater than $E_{C I} / e$ and $E_{I V} / e$ ), the IBSC breaks with the trade-off between current and voltage that governs SGSCs and can, therefore, achieve higher efficiencies.

Many attempts to manufacture an operational IBSC have been made in the past two decades, using different technological approaches to manufacture IB materials $[3,4]$. Early reports demonstrated the production of extra photocurrent via transitions 1 and $2[5,6]$ and the preservation of the voltage [7]. Since then, the IBSC working principles have been demonstrated using different IB materials (see Refs. [3,4] for a review). However, separately, neither the production of a below-band-gap photocurrent nor the preservation of the voltage actually demonstrates the complete IBSC operation. This is because photocurrent measurements are generally taken [5,8-11] under short-circuit conditions $(V=0)$, whereas the preservation of the voltage is demonstrated $[7,12]$ by measuring the open-circuit voltage $V_{O C}$ of the cell $(J=0)$. Hence, in both cases the electrical power $P=J \times V$ produced by the cell is zero. As the output voltage of the solar cell increases from zero, so does the recombination via the IB. For the IB to convey a benefit in the photovoltaic conversion of solar cells, there must be still a positive net photogeneration of carriers thanks to below-band-gap absorption at output voltages greater than both $E_{C I} / e$ and $E_{I V} / e$. Otherwise, the presence of the IB would have a null or negative impact in the efficiency of the solar cell. However, the demonstration that below-band-gap photons can be used to produce electrical work at voltages higher than their corresponding energies (divided by $e$ ) is lacking [4]. Few works [13-15] reported below-band-gap photocurrent under applied positive voltage bias and, in general, it was found that the photocurrent vanished as the bias increased. Simultaneous demonstration of below-band-gap photocurrent and voltage preservation is key to strengthening the validity of the IBSC framework because, as we shall show, it implies the existence of three distinct QFLs, a necessary condition for exceeding the efficiency limit of SGSCs. In this Letter, we demonstrate the production of electrical work due to below-band-gap absorption in an IBSC operating close to the maximum power point, providing the missing piece of confirmation of the IBSC theoretical framework. In this respect, we demonstrate a photocurrent response to the absorption of photons with an energy $E=$ $E_{C I}$ while the cell is operating at $V>E_{C I} / e>E_{I V} / e$, thus simultaneously verifying the two founding principles of the IBSC.

Quantum dot (QD) semiconductors embedded in a host are the most common technological approach for engineering IB materials [3]. Using this approach, the IB is formed by electronic states confined in the QD material whereas the $\mathrm{CB}$ and $\mathrm{VB}$ originate from the host semiconductor, as illustrated in Fig. 1(b). For this study, we use an IBSC (a)

(b)
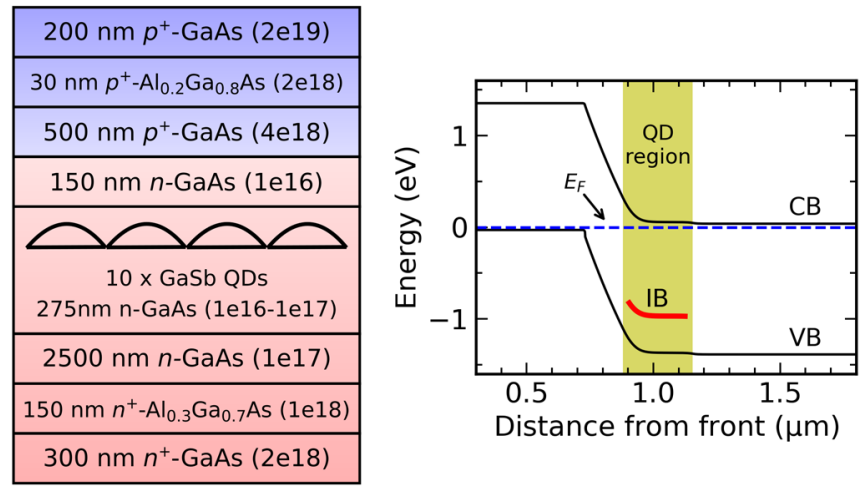

FIG. 2. (a) Layer structure of the $\mathrm{GaSb} / \mathrm{GaAs}$ QD-IBSC studied. Background colors illustrate the doping character of each layer: red for $n$ type, blue for $p$ type. (b) Calculated band diagram, at room temperature, of the sample studied. The Fermi level $E_{F}$ is set to the zero-energy value. The shadowed area highlights the QD region. The CB and VB have been calculated, using the PC1D software [19], for a structure similar to (a) but without QDs. The top edge of the IB (red line) lies in a quasi flat band region.

prototype containing 10 layers of $\mathrm{GaSb} / \mathrm{GaAs}$ QDs (GaSb QDs embedded in GaAs). Figure 2(a) details the simplified layer structure of the sample studied. Note that the QDs are placed outside the space charge region, for the reasons justified in Ref. [16], within an $n$-doped layer [see Fig. 2(b)], which results in full electron occupation of the IB. The semiconductor structure was grown in a molecular beam epitaxy reactor. The QD layers were formed using the Stranski-Krastanov growth mode. Metal was deposited on the top ( $p$ side) and bottom ( $n$ side) of the semiconductor structure to complete the solar cell. Full details of the sample growth and processing, together with transmission electron microscope images of the QD layers, are reported elsewhere [17,18].

For measurements, we placed the sample inside a closedcycle $\mathrm{He}$ cryostat equipped with $\mathrm{CaF}_{2}$ windows. The temperature $T$ of the sample was set to $9 \mathrm{~K}$. Figure 3 shows the $J-V$ characteristic of our sample under illumination with a $1.93 \mathrm{eV}$ diode laser. The excitation power density was approximately $7.8 \mu \mathrm{W} / \mathrm{cm}^{2}$ over a sample area of $6.3 \mathrm{~mm}^{2}$. The measured short-circuit current $J_{S C}$ is $1.59 \mu \mathrm{A} / \mathrm{cm}^{2}$ and the $V_{O C}$ is $1.236 \mathrm{~V}$. Under the excitation conditions employed, the theoretical maximum current generated (internal quantum efficiency of 1) is approximately $1.47 \mu \mathrm{A} / \mathrm{cm}^{2}$, after taking into account the reflection at the front interface between the air and GaAs (using 3.82 as the GaAs refractive index [20]) and considering that the photons absorbed in the $p$-GaAs contact layer do not contribute to the photocurrent. The electrical power produced by the cell reaches its maximum at a voltage in the vicinity of $1.15 \mathrm{~V}$.

Figure 4(a) shows the spectral photocurrent of our sample. The solar cell was illuminated with two photon 


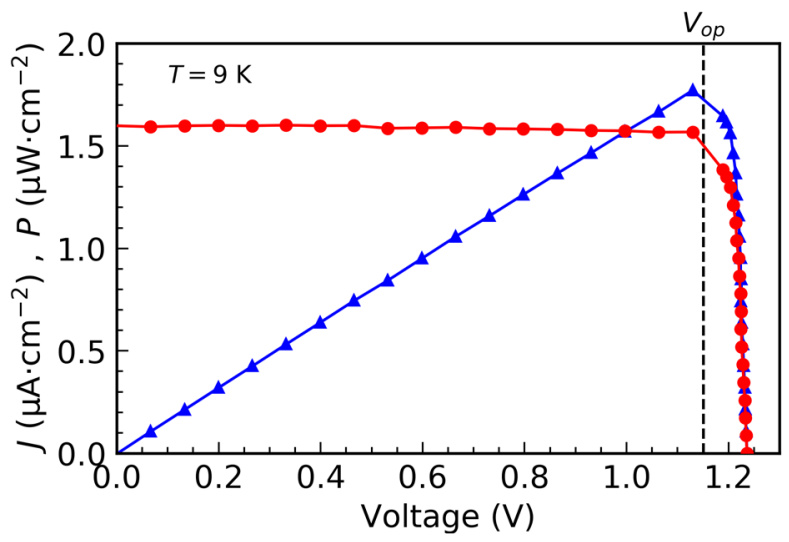

FIG. 3. Current-voltage curve (red circles) at $T=9 \mathrm{~K}$ of the $\mathrm{GaSb} / \mathrm{GaAs}$ QD-IBSC studied, under excitation with a continuous laser diode with a photon energy of $1.93 \mathrm{eV}$. The electrical power $P$ produced by the solar cell is plotted with blue triangles. $V_{o p}=1.15 \mathrm{~V}$ is the operating voltage at which the photocurrent measurement of Fig. 4(a) is taken.

beams [see Fig. 4(b)]. The first beam was the $1.93 \mathrm{eV}$ laser used for the $J-V$ measurements of Fig. 3 operating at the same pumping power. The purpose of this beam was to set steady-state light-bias conditions for the measurement. The second beam was monochromatic light whose energy could be swept in the $0.8-1.8 \mathrm{eV}$ range. For this, light from a quartz-tungsten-halogen (QTH) lamp was passed through a monochromator. The monochromatic light power reaching the sample was approximately $500 \mu \mathrm{W} / \mathrm{cm}^{2}$ at $0.8 \mathrm{eV}$ and decreased exponentially to $5 \mu \mathrm{W} / \mathrm{cm}^{2}$ at $1.8 \mathrm{eV}$. The recorded signal was the current response to the second beam. Detection was carried out using standard lock-in techniques including a low-noise current amplifier. The same amplifier was used to set a constant operating voltage $V_{o p}=1.15 \mathrm{~V}$ between the terminals of the solar cell so that it is operating at its maximum power point. Note that, in this configuration, the solar cell produces electrical work whereas the amplifier acts as a passive load, $R_{\text {load }}$, that fixes the desired operating point.

Figure 4(a) (red solid line) demonstrates production of electrical work due to below-band-gap absorption in an IBSC operating close to its maximum power point. Moreover, it demonstrates a photocurrent contribution for incident photons with $E=E_{C I}$ while the solar cell is operating at $e V_{o p}>E_{C I}$, thus simultaneously verifying the two basic pillars of IBSC operation. For comparison, we also show the photocurrent response to monochromatic light when the laser is OFF and the cell is short circuited (black dashed line). The addition of the laser and the consequent biasing to $V_{o p}$ give rise to a reduction of around a factor 2 in the photocurrent. We ascribe this effect to an increased recombination in the device when biased, which diminishes the collection efficiency of photogenerated carriers. The two photocurrent edges serve to identify $E_{C V} \approx 1.48$ and $E_{C I} \approx 1.02 \mathrm{eV}$. $E_{C I}$ is more precisely determined by electroluminescence (EL) spectra like the one shown in the inset. Two EL measurements were taken, using injection currents of 0.23 and $0.69 \mathrm{~A} \mathrm{~cm}^{-2}$, and yielding $E_{C I}$ equal to 1.017 and $1.022 \mathrm{eV}$, respectively, showing a blueshift with increasing bias. Following this tendency, it is likely that the actual value of $E_{C I}$ in the photocurrent measurements is slightly less than $1.02 \mathrm{eV}$. At $9 \mathrm{~K}$, the theoretical value of the GaAs band gap is approximately $1.52 \mathrm{eV}$, however, narrowing effects due to heavy doping $[21,22]$ could justify a value a few tens of meV smaller.

Three different energy regions can be identified in the photocurrent spectrum. For $E>E_{C V}$, the photocurrent is due to absorption via transition 3 [see Fig. 1(b)]. For $E_{C V}>E>E_{C I}$, the photocurrent is due to absorption via transition 2. In this region, the photocurrent is lower $\left(\sim 10^{-8} \mathrm{~A} \mathrm{~cm}^{-2}\right)$ because of the reduced absorption in (a)

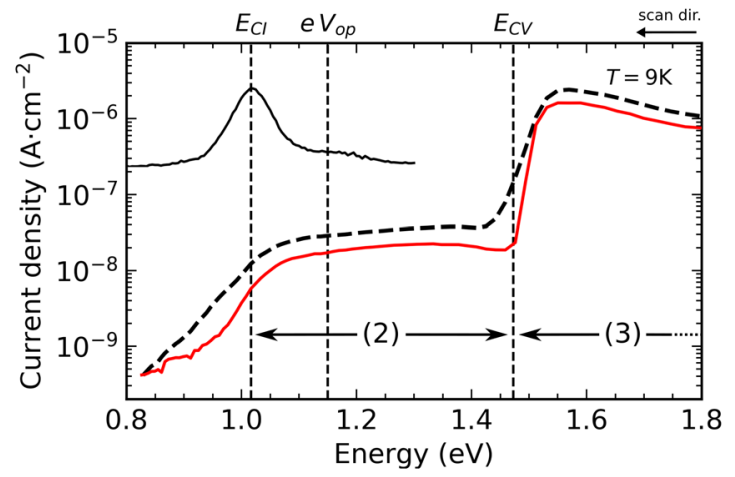

(b)

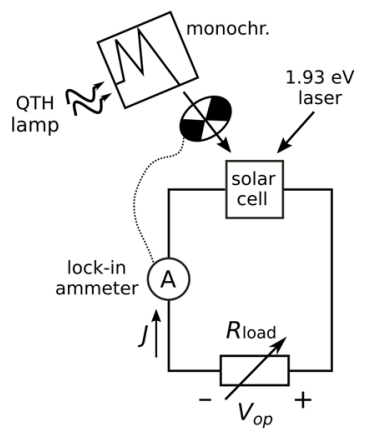

FIG. 4. (a) Spectral photocurrent of the GaSb/GaAs QD-IBSC studied. Red solid line: measurement with light bias (1.93 eV laser) using the setup described in (b). Black dashed line: conventional photocurrent measurement at short circuit $\left(R_{\text {load }}=0 \Omega\right)$ and without light bias. The energy regions corresponding to response to transitions 2 and 3 are also indicated. The inset shows the EL spectrum of the sample for an injection current of $0.23 \mathrm{~A} \mathrm{~cm}^{-2}$. All measurements are taken at $9 \mathrm{~K}$. (b) Diagram of the spectral photocurrent setup used for measuring (a) as described in the main text. 
the GaSb QDs as compared to the bulk GaAs. This low value of photocurrent means that, during the measurement, the solar cell is moving only slightly around the working point set by the laser $\left(\sim 10^{-6} \mathrm{~A} \mathrm{~cm}^{-2}\right)$, so it can be considered that it remains at the maximum power point. For $E<E_{C I}$, the photocurrent drops steadily as does the absorption in the QDs. Photocurrent due to absorption via transition 1 (not shown) is almost negligible (see Ref. [17]). The presence of photocurrent via transition 2 (IB $\rightarrow \mathrm{CB}$ absorption) and the absence of photocurrent via transition 1 (VB $\rightarrow$ IB absorption) are in agreement with a fully occupied IB as a consequence of the $n$-doping character of the QD region [Fig. 2(b)].

In Fig. 4(a), we have measured the current response to a monochromatic beam with $E_{C I}<E<E_{C V}$ intended to trigger the IB $\rightarrow \mathrm{CB}$ transition [see Fig. 1(b)]. However, the VB $\rightarrow$ IB transition should, in principle, take place as well so that electron-hole pairs are generated in the $\mathrm{CB}$ and $\mathrm{VB}$, and the current can be extracted. Figure 5 illustrates six different processes that could account for the production of photocurrent under monochromatic illumination with $E_{C I}<E<E_{C V}$, as discussed in the following. (a) Tunneling [23] between the VB and the IB: This process is unwanted in an IBSC because it prevents the splitting of the QFLs of the two bands involved [15]. Because the QDs in our sample are in a quasi-flat-band region [Fig. 2(b)], in contrast to previous works [13-15], (a)

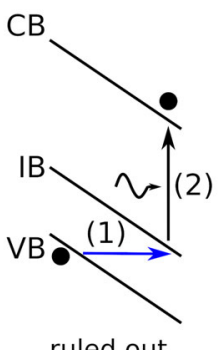

ruled out

(d)

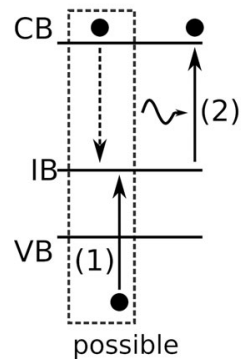

(b)

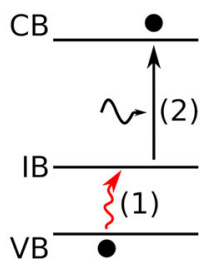

ruled out

(e)

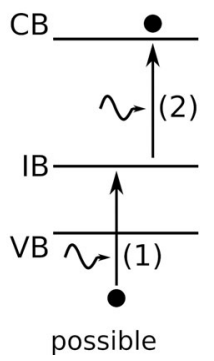

(c)

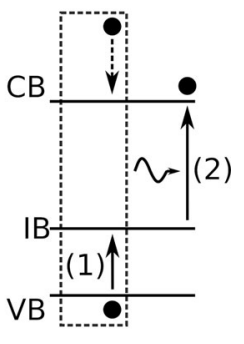

ruled out

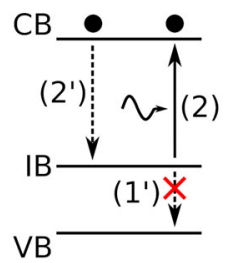

ruled out

FIG. 5. Processes that could explain the photocurrent measured in Fig. 4(a) under monochromatic illumination with photons of an energy $E_{C I}<E<E_{C V}$. (a) Tunneling. (b) Thermal excitation. (c) and (d) Auger generation. (e) Photon absorption. (f) Reduced $\mathrm{CB} \rightarrow \mathrm{IB} \rightarrow \mathrm{VB}$ recombination.

tunneling should be very inefficient. (b) The thermal excitation of carriers [23] into the IB: This process also is undesired because it also prevents QFL splitting [7]. Our measurements are taken at $9 \mathrm{~K}$, so this mechanism would also be very inefficient. (c) Auger generation of carriers from the VB into the IB by taking energy from excited carriers in the $\mathrm{CB}$ (or the VB). However, at $9 \mathrm{~K}$, the concentration of excited carriers with the required extraenergy $\left(E_{I V} \approx 0.49 \mathrm{eV}[17,18]\right)$ above their band edge is very low because, after photogeneration, the carriers will thermalize until the carrier population follows Fermi-Dirac statistics. Hence, we consider this process to be very unlikely. (d) Auger generation of carriers from the VB into the IB via deexcitation of carriers in the $\mathrm{CB}$ back to the IB [24]. (e) Photon absorption: Incident photons may trigger both the $\mathrm{IB} \rightarrow \mathrm{CB}$ and the $\mathrm{VB} \rightarrow \mathrm{IB}$ transitions, if the absorption coefficients of these transitions overlap [25]. (f) Reduced CB $\rightarrow$ IB $\rightarrow$ VB recombination: In the measurement with light bias, there is a constant offset of photocurrent flowing through the device. At $V_{o p}$, a fraction of the electron-hole pairs generated by this beam may undergo recombination in the QDs via the IB. It would be possible that the second monochromatic beam pumps electrons back from the IB to the $\mathrm{CB}$, thus preventing IB $\rightarrow$ $\mathrm{VB}$ relaxation and reducing the overall $\mathrm{CB} \rightarrow \mathrm{IB} \rightarrow \mathrm{VB}$ recombination, which would increase the current flow [26]. However, this process cannot take place in the measurement without light bias [Fig. 4(a), dashed line], so we deduce that it is not responsible for the measured current.

The IBSC theoretical framework requires the definition of 3 QFLs to preserve the output voltage while harvesting below-band-gap photons. With the result in Fig. 3 we have demonstrated $e V>E_{C I}$ or, equivalently, $\mu_{C}-\mu_{V}>E_{C I}$. We will now prove that the result in Fig. 4(a) demonstrates the existence of at least one QFL $\mu_{I}$ associated to the IB such that $\mu_{I} \neq \mu_{V}$ and $\mu_{C}-\mu_{I}<E_{C I}$. We will rely on the band diagrams detailed in Fig. 6 for our analysis. Figure 6(a) shows the case of only two QFLs (or $\mu_{I}=\mu_{V}$ ). $\mu_{C}$ sits close to the bottom edge of the CB because the QDs (a)

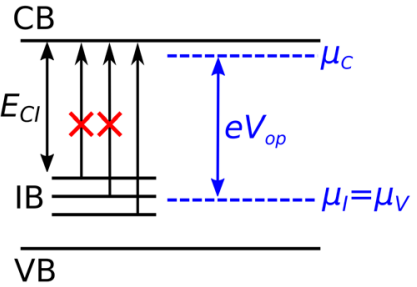

(b)

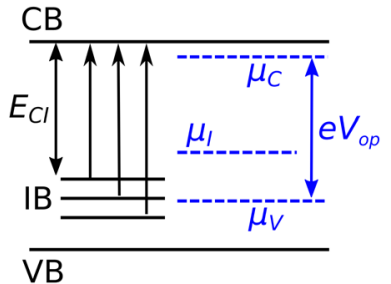

FIG. 6. Electronic bands and their associated QFLs at $e V_{o p}>$ $E_{C I}$ in the $\mathrm{GaSb} / \mathrm{GaAs}$ QD-IBSC studied, for the case of (a) $\mu_{I}=\mu_{V}$ and (b) $\mu_{I} \neq \mu_{V}$. Vertical arrows indicate belowband-gap photon absorption via transition 2. In (a) red crosses illustrate prevented absorption owing to the lack of electrons in the IB states with an energy greater than $\mu_{I}$. 
are in an $n$ region [Fig. 2(b)]. When the bias is such that $e V>E_{C I}, \mu_{V}$ lies below the top edge of the IB. In this situation, the IB states with an energy greater than $\mu_{V}$ are virtually empty (remember that $T=9 \mathrm{~K}$ ). This would mean that absorption of photons with an energy in the vicinity of $E_{C I}$ is no longer possible, and hence neither is photo-response. However, this is not consistent with the photocurrent spectra in Fig. 4(a), which leads us to conclude that the assumption of two QFLs is not correct. Therefore, there must be at least a distinct QFL $\mu_{I} \neq \mu_{V}$ [Fig. 6(b)] such that $\mu_{C}-\mu_{I}<E_{C I}=1.02 \mathrm{eV}$ when $V=V_{o p}=1.15 \mathrm{~V}$, so that the most energetic IB states are still populated, and absorption via transition 2 can take place.

In previous reports [13-15], the below-band-gap photocurrent eventually vanished upon forward voltage biasing at values less than the threshold of voltage preservation. This was indicative of only two QFLs describing the carrier populations in the device, as previously discussed. Specific experiments intended to verify the existence of 3 QFLs in QD-IBSCs have been previously carried out. Ref. [27] reported 3 QFLs at room temperature in InAs/GaAs QD-IBSCs through analysis of quantum efficiency and EL measurements, but it was later shown that those results were probably altered by sample heating [28]. Analyses of the temperature dependence of the $V_{O C}$ pointed towards the absence of a split between $\mu_{I}$ and $\mu_{V}$ in $\mathrm{Ge} / \mathrm{Si}$ [29], and $\mu_{I}$ and $\mu_{C}$ in $\mathrm{InP} / \mathrm{InGaP}$ [30] QD-IBSCs. Outside the QD-based IBSC, three emission peaks in low-temperature EL experiments were reported in a GaNAs alloy prototype [31], in agreement with the existence of 3 QFLs. However, in the EL experiment, the solar cell is consuming power instead of producing it as it does under proper operation. Our results demonstrate the existence of 3 QFLs in a QD-based IBSC under illumination operating at the maximum power point, beyond the threshold of voltage preservation. This result represents experimental evidence of the consistency of the IBSC theoretical framework.

In summary, we have demonstrated that below-band-gap photons can be used to produce nonzero electrical work in an IBSC without imposing a fundamental limit to the voltage, providing the missing piece of evidence that completes the IBSC theoretical framework. To this end, it was necessary to suppress any thermal and tunnelingassisted carrier escape processes, which prevent QFL splitting. The experiment of spectral photocurrent under light-biasing described herein is a valid tool for discriminating IB materials and IBSC structures with the potential to achieve high efficiency. We have shown that the reported results imply the existence of a third distinct QFL associated to the IB. The demonstration of the existence of three QFLs in an IBSC while it is producing electrical work represents yet another verification of the validity of the IBSC theoretical framework.
We would like to acknowledge the anonymous referees for providing insights regarding processes (d) and (f) of Fig. 5 during the review process. This work was supported in part by the Project 2GAPS (TEC2017-92301-EXP) funded by the Spanish Ministerio de Ciencia, Innovación y Universidades, and the Project MADRID-PV2-CM (P2018/EMT-4308) funded by the Comunidad de Madrid supported with FEDER funds. The materials growth and device manufacture at the University of Michigan was supported as part of the Center for Solar and Thermal Energy Conversion, an Energy Frontier Research Center funded by the U.S. Department of Energy, Office of Science, Office of Basic Energy Sciences under Award No. DE-SC0000957.

*i.ramiro@ies.upm.es, he/him/his

[1] W. Shockley and H. J. Queisser, Detailed balance limit of efficiency of p n junction solar cells, J. Appl. Phys. 32, 510 (1961).

[2] A. Luque and A. Martí, Increasing the Efficiency of Ideal Solar Cells by Photon Induced Transitions at Intermediate Levels, Phys. Rev. Lett. 78, 5014 (1997).

[3] I. Ramiro, A. Martí, E. Antolín, and A. Luque, Review of experimental results related to the operation of intermediate band solar cells, IEEE J. Photovoltaics 4736 (2014).

[4] I. Ramiro and A. Martí, Intermediate band solar cells: Present and future, Prog. Photovoltaics (2020).

[5] A. Luque, A. Martí, C. Stanley, N. López, L. Cuadra, D. Zhou, J. L. Pearson, and A. McKee, General equivalent circuit for intermediate band devices: Potentials, currents and electroluminescence, J. Appl. Phys. 96, 903 (2004).

[6] A. Martí, E. Antolín, C. R. Stanley, C. D. Farmer, N. Lopez, P. Díaz, E. Canovas, P. G. Linares, and A. Luque, Production of Photocurrent due to Intermediate-toConduction-Band Transitions: A Demonstration of a Key Operating Principle of the Intermediate-Band Solar Cell, Phys. Rev. Lett. 97, 247701 (2006).

[7] E. Antolín, A. Martí, P. G. Linares, I. Ramiro, E. Hernández, C. D. Farmer, C. R. Stanley, and A. Luque, Advances in quantum dot intermediate band solar cells, 35th IEEE PVSC (2010).

[8] S. A. Blokhin, A. V. Sakharov, A. M. Nadtochy, A. S. Pauysov, M. V. Maximov, N. N. Ledentsov, A. R. Kovsh, S. S. Mikhrin, V. M. Lantratov, and S.A. Mintairov, AlGaAs/GaAs photovoltaic cells with an array of InGaAs QDs, Semiconductors 43, 514 (2009).

[9] R. Oshima, A. Takata, and Y. Okada, Strain-compensated InAs/GaNAs quantum dots for use in high-efficiency solar cells, Appl. Phys. Lett. 93, 083111 (2008).

[10] T. Sugaya, O. Numakami, R. Oshima, S. Furue, H. Komaki, T. Amano, K. Matsubara, Y. Okano, and S. Niki, Ultra-high stacks of InGaAs/GaAs quantum dots for high efficiency solar cells, Energy Environ. Sci. 5, 6233 (2012).

[11] C. G. Bailey, D. V. Forbes, S. J. Polly, Z. S. Bittner, Y. Dai, C. Mackos, R. P. Raffaelle, and S. M. Hubbard, Open-circuit voltage improvement of InAs/GaAs quantum-dot solar cells using reduced InAs coverage, IEEE J. Photovoltaics 2, 269 (2012). 
[12] P. G. Linares, A. Martí, E. Antolín, C. D. Farmer, Í. Ramiro, C. R. Stanley, and A. Luque, Voltage recovery in intermediate band solar cells, Sol. Energy Mater. Sol. Cells 98, 240 (2012).

[13] D. Alonso-Álvarez, B. Alén, J. M. García, and J. M. Ripalda, Optical investigation of type II GaSb/GaAs self-assembled quantum dots, Appl. Phys. Lett. 91, 263103 (2007).

[14] Y. Dai, S. Polly, S. Hellstroem, D. V. Forbes, and S. M. Hubbard, Electric field effect on carrier escape from InAs/GaAs quantum dots solar cells, 40th IEEE PVSC (2014).

[15] I. Ramiro, E. Antolín, A. Martí, C. D. Farmer, C. R. Stanley, and A. Luque, Experimental demonstration of the effect of field damping layers in quantum-dot intermediate band solar cells, Sol. Energy Mater. Sol. Cells 140, 299 (2015).

[16] A. Martí, E. Antolín, E. Cánovas, N. López, P. G. Linares, A. Luque, C. R. Stanley, and C. D. Farmer, Elements of the design and analysis of quantum-dot intermediate band solar cells, Thin Solid Films 516, 6716 (2008).

[17] I. Ramiro, E. Antolín, J. Hwang, A. Teran, A. J. Martin, P. G. Linares, J. Millunchick, J. Philips, A. Martí, and A. Luque, Three-bandgap absolute quantum efficiency in $\mathrm{GaSb} / \mathrm{GaAs}$ quantum dot intermediate band solar cells, IEEE J. Photovoltaics 7, 508 (2017).

[18] I. Ramiro, J. Villa, C. Tablero, E. Antolín, A. Luque, A. Martí, J. Hwang, J. Phillips, A. J. J. Martin, and J. Millunchick, Analysis of the intermediate-band absorption properties of type-II GaSb/GaAs quantum-dot photovoltaics, Phys. Rev. B 96, 125422 (2017).

[19] P. A. Basore, PC1D, https://www.engineering.unsw.edu.au/ energy-engineering/research/software-data-links/pc1d-softwarefor-modelling-a-solar-cell.

[20] D. E. Aspnes, S. M. Kelso, R. A. Logan, and R. Bhat, Optical properties of $\mathrm{Al}_{x} \mathrm{Ga}_{1-x} \mathrm{As}$, J. Appl. Phys. 60, 754 (1986).

[21] G. Borghs, K. Bhattacharyya, K. Deneffe, P. Van Mieghem, and R. Mertens, Band-gap narrowing in highly doped n-and p-type GaAs studied by photoluminescence spectroscopy, J. Appl. Phys. 66, 4381 (1989).
[22] S. C. Jain, J. M. McGregor, D. J. Roulston, and P. Balk, Modified simple expression for bandgap narrowing in N-Type GaAs, Solid State Electron. 35, 639 (1992).

[23] P. W. Fry, I. E. Itskevich, S. R. Parnell, J. J. Finley, L. R. Wilson, K. L. Schumacher, D. J. Mowbray, M. S. Skolnick, M. Al-Khafaji, A. G. Cullis, M. Hopkinson, J. C. Clark, and G. Hill, Photocurrent spectroscopy of InAs/GaAs self-assembled quantum dots, Phys. Rev. B 62, 16784 (2000).

[24] A. Luque, A. Martí, and L. Cuadra, Impact-ionizationassisted intermediate band solar cell, IEEE Trans. Electron Devices 50, 447 (2003).

[25] L. Cuadra, A. Martí, and A. Luque, Influence of the overlap between the absorption coefficients on the efficiency of the intermediate band solar cell, IEEE Trans. Electron Devices 51, 1002 (2004).

[26] I. Ramiro, A. Martí, E. Antolín, E. López, A. Datas, A. Luque, J. M. Ripalda, and Y. González, Optically triggered infrared photodetector, Nano Lett. 15, 224 (2015).

[27] A. Luque, A. Martí, N. López, E. Antolín, E. Canovas, C. Stanley, C. Farmer, L. J. Caballero, L. Cuadra, and J. L. Balenzategui, Experimental analysis of the quasi-fermi level split in quantum dot intermediate-band solar cells, Appl. Phys. Lett. 87, 083505 (2005).

[28] A. A. Abouelsaood, M. Y. Ghannam, and J. Poortmans, On the reported experimental evidence for the quasi-fermi level split in quantum-dot intermediate-band solar cells, Prog. Photovoltaics 21, 209 (2013).

[29] T. Tayagaki, Y. Hoshi, and N. Usami, Investigation of the open-circuit voltage in solar cells doped with quantum dots, Sci. Rep. 3, 2703 (2013).

[30] T. Aihara, T. Tayagaki, Y. Nagato, Y. Okano, and T. Sugaya, Investigation of the open-circuit voltage in wide-bandgap InGaP-host InP quantum dot intermediate-band solar cells, Jpn. J. Appl. Phys. 57, 04FS04 (2018).

[31] N. López, L. A. Reichertz, K. M. Yu, K. Campman, and W. Walukiewicz, Engineering the Electronic Band Structure for Multiband Solar Cells, Phys. Rev. Lett. 106, 028701 (2011). 